\title{
Elevated Temperature Deformation Mechanisms in $\mathrm{Ta}_{2} \mathrm{C}$
}

\author{
N. De Leon,* B. Wang, ${ }^{*}$ C.R. Weinberger,** and G.B. Thompson* \\ * Department of Metallurgical and Materials Engineering, The University of Alabama, Box 870202, \\ Tuscaloosa, AL 35487-0202 \\ ** Sandia National Laboratories, Albuquerque, NM 87185-1411
}

The tantalum carbides are a class of ultrahigh melting temperature materials. An interesting property of tantalum carbides is the duality of mechanical responses with temperature; they exhibit classical ceramic brittle behavior at low temperatures but significant plasticity at elevated temperatures [1], as shown in Figure 1(a). While the cubic $\mathrm{TaC}$ phase, which has the $\mathrm{B} 1$ structure, has had its slip systems studied and defined [2], the hexagonal like $\alpha-\mathrm{Ta}_{2} \mathrm{C}$ phase, with its $\mathrm{CdI}$ antitype structure Figure 1(b), has not been extensively investigated. This paper addresses the thermo-mechanical deformation mechanisms in $\alpha-\mathrm{Ta}_{2} \mathrm{C}$. The plastic deformation mechanisms have been characterized using dynamical diffraction transmission electron microscopy (TEM) and high resolution transmission electron microscopy (HRTEM) techniques.

The $\alpha-\mathrm{Ta}_{2} \mathrm{C}\left(\mathrm{Ta}_{68} \mathrm{C}_{32}\right)$ specimens were prepared by mixing constitute $\mathrm{TaC}$ and $\mathrm{Ta}$ powder at the appropriate weight fractions and subjected to Hot Isostatic Pressing (HIP) at $200 \mathrm{MPa}$ in an argon atmosphere at $1600^{\circ} \mathrm{C}$. The HIP specimens was then machined into $3 \mathrm{~mm} \times 4 \mathrm{~mm} \times 45 \mathrm{~mm}$ bars and four-point flexural tested at approximately $1930^{\circ} \mathrm{C}$ in a graphite furnace under flowing Argon according to the modified specifications provided in MIL-STD-1942A. Post-loading, cross-section TEM foils were taken from the central region of the test bar where maximum deflection occurred. Xray diffraction (XRD) using a Bruker Discovery D8 General Area Diffraction System and Cu$\mathrm{K}_{\alpha}$ radiation confirmed that the specimen was $\alpha-\mathrm{Ta}_{2} \mathrm{C}$ pre- and post-mechanical testing. TEM foils were prepared using standard grinding, dimpling and ion milling to achieve electron transparency. The foil was characterized in a FEI F20 Tecnai (S)TEM and $\mathrm{C}_{\mathrm{s}}$-aberration corrected FEI Titan TEM.

Two major deformation mechanisms were identified: dislocation slip and stacking faults. Dense dislocation networks or forests were clearly visible in grains when viewed down the [0001] zone axis, Figure 1(c). Both basal and non-basal slip was observed as well as a Frank-Reed source for dislocation generation, Figure 1(d). The non-basal Burger's vector was determined to be a/3 [2113]. $\mathrm{Ta}_{2} \mathrm{C}$ 's c/a ratio of 1.59 [3] (ideal c/a is 1.63) makes it a candidate for non-basal plane slip because the basal plane is no longer the most densely packed plane. Stacking faults were observed edge-on from the $[11 \overline{2} 0\rfloor$ zone axis. This confirmed predominate faulting was on the basal plane. HRTEM micrographs, Figure 2(a), revealed that these faults were between the Ta-Ta bonds and not the Ta-C bonds, as shown in the crystal diagram of Figure 1(b). Using first principle calculations, the generalized stacking fault energy (GSFE) between the Ta-Ta and Ta-C basal planes were calculated to be an order of magnitude different in energy, Figure 2(b). This confirmed that the local metallic bond character provided for easier deformation accommodation.

\section{References:}

[1]A. Kelly and D.J. Rowcliffe, Journal of the American Ceramic Society 50(5) pp.253-256, 1967.

[2] C. Kim, G. Gottstien, and D.S. Grummon, Acta metal. mater. 42(7), pp. 2291-2301, 1994.

[3] E. K. Storms “The Tantalum-Tantalum Carbide System” in The Refractory Carbides, Academic Press, 1967. 
[4] This work has been supported under ARO-W911NF-08-1-0300. The authors thank L.A. Matson, R. Wheeler, and P. Mogilevsky, K. Mahalingam for assistance in use and access of the AFRL Titan TEM for the HRTEM micrographs.
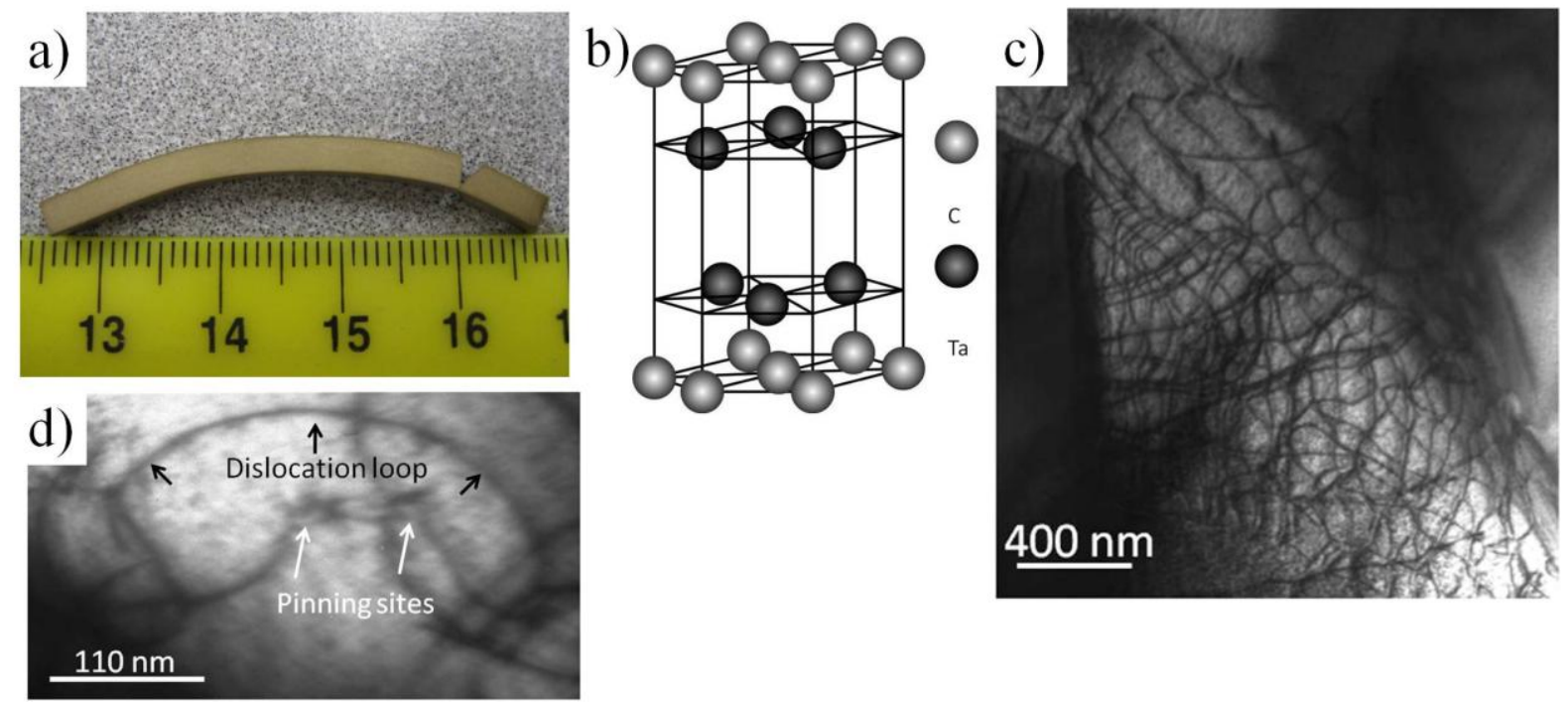

FIG. 1. a) Photograph of a $\mathrm{Ta}_{2} \mathrm{C}$ specimen post 4-point bend mechanical tested at $1930^{\circ} \mathrm{C}$. Note the significant plastic deformation. b) The hexagonal $\mathrm{Ta}_{2} \mathrm{C}$ phase. c) Bright Field $\mathrm{TEM}$ micrographs which exhibit a dense dislocation network. d) Frank-Read source as viewed near the [0001] zone axis.

a)

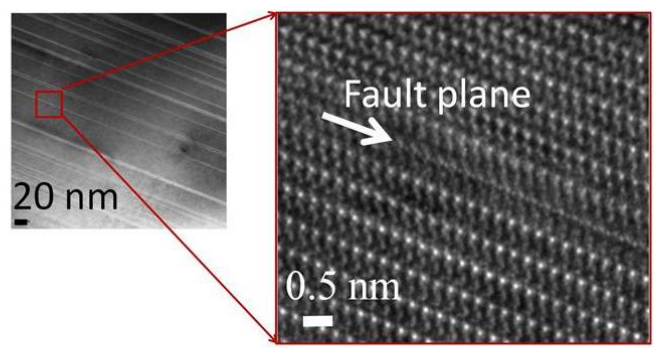

b)
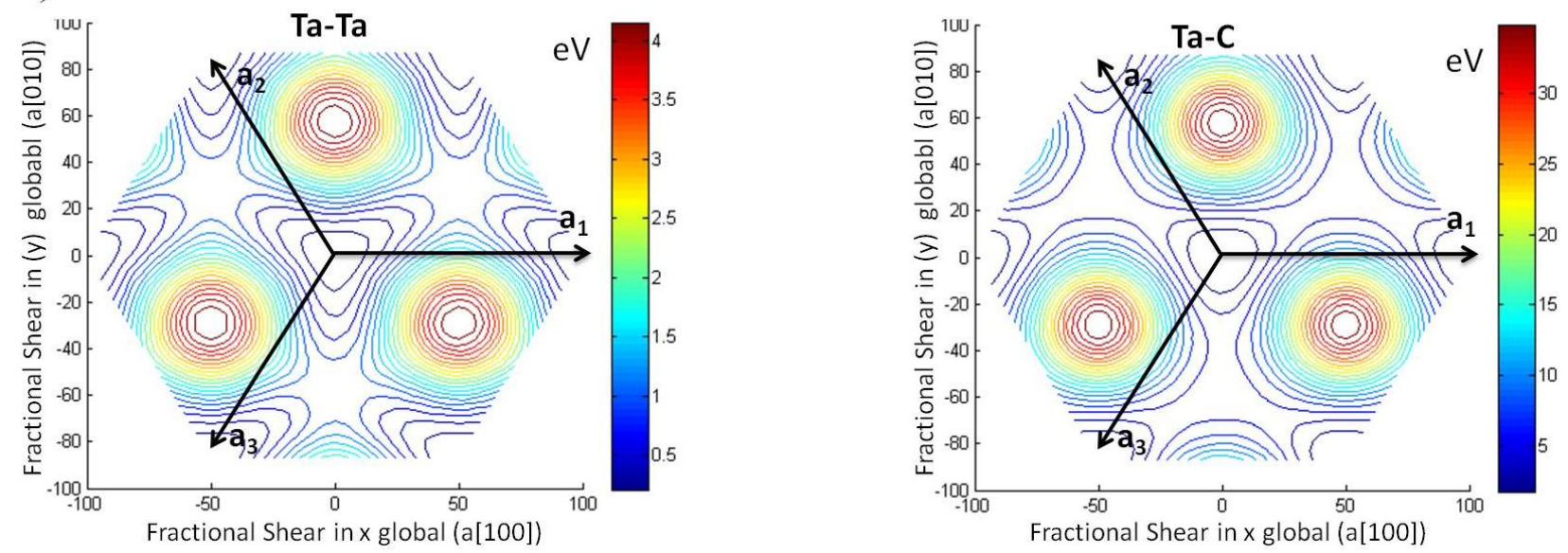

FIG. 2. a) HRTEM image showing the stacking fault as viewed down the $[1 \overline{1} 0\rfloor$ zone axis. b) GSFE calculations on the basal plane for the Ta-Ta and Ta-C showing the order of magnitude difference in energy requirements, $\sim 3.5 \mathrm{eV}$ vs. $\sim 30 \mathrm{eV}$ respectively. 